\title{
Avaliação de impactos ambientais gerados na implantação e operação de subestação de energia elétrica: um estudo de caso em Palhoça, SC
}

\author{
Evaluation of environmental impacts generated by the implementation and operation of an \\ electrical substation: a case study in Palhoça, SC
}

\section{Eliana Turmina ${ }^{1 *}$, Maria Raquel Kanieski ${ }^{1}$, Larissa Antunes de Jesus ${ }^{1}$, Luiara Heerdt da Rosa ${ }^{2}$, Lais Gervasio Batista $^{2}$ \& Alexandre Nascimento de Almeida ${ }^{3}$}

\author{
${ }^{1}$ Universidade do Estado de Santa Catarina, Lages, SC, Brasil. *Autor para correspondência: eliana_turmina@hotmail.com \\ ${ }^{2}$ Geoconsultores Engenharia e Meio Ambiente, Tubarão, SC, Brasil. \\ ${ }^{3}$ Universidade de Brasília, Brasília, DF, Brasil.
}

Submissão: 15/09/2017 / Aceite: 13/06/2018

\begin{abstract}
RESUMO
A energia elétrica é fundamental nos dias atuais, mas para chegar até empreendimentos agroveterinários, bem como ao consumidor final, há necessidade da implantação de empreendimentos que podem impactar o meio ambiente. Neste sentido, a avaliação de impactos ambientais desempenha um importante papel, contribuindo para que o desenvolvimento econômico e social estejam aliados aos fatores ambientais. Este estudo tem como objetivo avaliar por meio do método Ad Hoc, bem como de ambas as matrizes de interação e ponderação de atributos os impactos gerados por uma subestação de energia elétrica, situado no município de Palhoça, Santa Catarina. Foram analisadas as atividades relacionadas às etapas de implantação do empreendimento que consistem na terraplanagem, quando necessário e, posteriormente, as atividades de construção civil. Finalmente, a etapa de operação do empreendimento que consiste na identificação das ações geradoras que são as atividades de terraplanagem, locação e construção de estruturas, escavações, sistemas de drenagem e aterramento, montagens eletromecânicas, mobilização de pessoal e manutenção da subestação e consequentemente os impactos ambientais. As atividades realizadas pelo empreendimento em questão que podem afetar de forma direta os aspectos ambientais como: água, solo, emissões atmosféricas, entre outras, além de aspectos sociais. Com a utilização das matrizes de ponderação e interação, foi possível diagnosticar que a maioria das atividades impactantes ocorre na fase de implantação do empreendimento. A maioria dos impactos, para o empreendimento selecionado, foi classificado como de pequena importância, baixa magnitude e reversíveis. Este estudo de caso poderá auxiliar futuras pesquisas e estudos ambientais nas mais diversas áreas, de modo a apresentar um método de demonstrar a importância e interação de cada impacto, além de evidenciar para os órgãos licenciadores um método alternativo o qual pode ser exigido para os futuros estudos de impacto ambiental.
\end{abstract}

PALAVRAS-CHAVE: transmissão de energia, empreendimento impactante, matriz.

\begin{abstract}
Electricity is essential nowadays, but to reach agro-veterinary enterprises, as well as the final consumer, there is a need to implement projects that can impact the environment. As such, evaluating environmental impacts plays an important role, contributing to economic and social development allied to environmental factors. The objective of this study was to evaluate the impacts generated by an electrical substation, located in the municipality of Palhoça, state of Santa Catarina, by means of the Ad Hoc method, as well as of both matrices of interaction and attribute weighting. We analyzed the activities related to the implementation stages of the project consisting of earthworks when necessary and, later, civil construction activities. Finally, the operation stage of the enterprise consists of identifying the generating actions that are the activities of earthworks, leasing and construction of structures, excavations, drainage and grounding systems, electromechanical assemblies, personnel mobilization and substation maintenance and, consequently, environmental impacts. The activities conducted by the enterprise in question that can directly affect the environmental aspects such as: water, soil, atmospheric emissions, among others, besides social aspects. Using weighting and interaction matrices, it was possible to diagnose that most impacting activities occur in the implementation phase of the project. Most impacts, for the selected enterprise, were classified as of small importance, low magnitude and reversible. This case study may
\end{abstract}


support future research and environmental studies in a variety of areas, depicting a method to demonstrate the importance and interaction of each impact, as well as presenting to licensing agencies an alternative method which may be required for future environmental impact studies.

KEYWORDS: power transmission, impactful enterprise, matrix.

\section{INTRODUÇÃO}

Atualmente, há uma crescente demanda por geração de energia elétrica, sendo que esta pode ser considerada uma das principais constituintes da sociedade contemporânea, desempenhando um papel fundamental no crescimento econômico. No setor agroveterinário a energia elétrica é muito importante para a produção agrícola, entre outras atividades pertinentes ao ramo. Mas para que haja geração de energia, é necessária a construção de empreendimentos que, como consequência, podem gerar impactos negativos ao meio ambiente como um todo, sendo que grande parte da energia elétrica gerada no Brasil, provém das hidrelétricas.

Um dos empreendimentos necessários utilizados nesse processo de geração de energia, o qual é responsável pelo transporte da energia elétrica das usinas, são as linhas de transmissão (LT), existentes em todo o país. No sistema de transferência há o envolvimento de condutores e equipamentos, com diferentes distâncias e largura de corredores, formas e níveis de tensão, interligando as usinas e consumidores, o que faz com que a energia elétrica produzida possa ser utilizada (PIRES 2005).

Porém, o sistema de transmissão de energia elétrica não se trata apenas das LT's, mas, engloba toda rede que interliga as usinas geradoras às subestações da rede de distribuição, no qual, o Brasil encontra-se quase que totalmente interligado. A energia elétrica é transportada de um ponto transmissor a um terminal receptor (linhas de transmissão e linhas de distribuição de energia elétrica) (RODRIGUES 2014).

De acordo com LIMA (2008), a subestação de energia elétrica é o elo final da transmissão, com o rebaixamento da tensão e o início da distribuição. Classificam-se quanto à função, ao sistema, ao nível de tensão, instalação e ao tipo de isolamento. DINIZ (2003) acrescenta que as subestações são instalações que recebem energia elétrica em alta voltagem, convertendo para baixa, com a finalidade de distribuir para a população. As estruturas que fazem esta conversão são os transformadores de energia elétrica, sendo as principais fontes de ruído nas subestações.

Ainda, PIRES (2005) ressalta que os sistemas de transmissão de energia elétrica, apesar de apresentarem pontos positivos importantes para a sociedade, podem causar distúrbios no meio ambiente ao longo de seus percursos e nas áreas que são implantadas. Neste contexto se faz necessária uma avaliação de impactos ambientais. Para RODRIGUES (2014), avaliação do impacto ambiental é uma estratégia com intuito de garantir a sustentabilidade econômica, social e ambiental.

Conforme a Resolução do CONAMA № 06 de 1987, no licenciamento de subestações e linhas de transmissão, a licença prévia deve ser requerida no início do planejamento do empreendimento, antes de definida sua localização ou caminhamento definitivo. Dando sequência, faz necessária a licença de instalação, que acontece após a conclusão do projeto executivo e antes do início das obras. Por último, requer a licença de operação, que será antes da entrada em operação comercial (BRASIL 1987).

Acrescenta-se que de acordo com a Resolução do CONAMA no 279 de 2001, os sistemas de transmissão de energia elétrica (linhas de transmissão e subestações), quando estes forem empreendimentos com impacto ambiental de pequeno porte, aplica-se o licenciamento ambiental simplificado (BRASIL 2001).

Para PIRES (2005), em termos de impactos ambientais, as subestações equivalem a qualquer planta industrial, pois afetam o uso do solo, antes mesmo da construção, no processo de aquisição do terreno. Os impactos que ocorrem durante a construção devem-se à movimentação do solo, retirada da cobertura vegetal, descarte incorreto dos resíduos líquidos e sólidos e também pelas intervenções nos equipamentos sociais, áreas comunitárias e locais de interesse histórico e cultural.

Segundo CAMARGO et al. (2004) os impactos ocasionados pela implantação de um empreendimento de geração de energia elétrica são definitivos e irreversíveis. No entanto, durante a fase de projeto, são definidos programas de mitigação ou compensação que são implantados no decorrer da construção e operação da usina. Já os impactos que são causados pela operação, são geralmente permanentes e contínuos, por isso devem ser monitorados e medidos. Para SILVA (2006) é essencial que sejam norteadas as ações das empresas, nas fases de planejamento, implantação e operação dos seus empreendimentos, pois a empresa empreendedora utiliza recursos naturais e desenvolve atividades que afetam o meio 
ambiente.

Conforme PIRES (2005) as subestações, quando não são enclausuradas, ocasionam impactos visuais, sendo que a concentração de estruturas e condutores, bem como, chegadas de linhas são as ações que impõem impacto negativo. A magnitude deste impacto relaciona-se com seu simbolismo e percepção, podendo variar com relação ao nível de renda e de escolaridade da população da área onde está inserida a subestação.

Neste contexto, este estudo teve por objetivo identificar e avaliar os impactos ambientais gerados com a instalação de subestação de energia localizado no Município de Palhoça, SC, com os métodos $A d$ Hoc, matriz de interação e matriz de ponderação, sendo estes métodos alternativos de matrizes usuais por empresas de consultoria.

\section{MATERIAL E MÉTODOS}

O empreendimento está previsto para ser implantado no município de Palhoça, região metropolitana de Florianópolis, no litoral do Estado de Santa Catarina, localizado na Bacia do Rio Cubatão Sul, inserido no bioma Mata Atlântica, Floresta Ombrófila Densa (FOD) na formação de terras baixas (IBGE 2012). A subestação será contígua à subestação Palhoça (Figura 1), se tratando de uma ampliação na porção norte da subestação existente, em um terreno de aproximadamente $3.300 \mathrm{~m}^{2}$.

Para a instalação e funcionamento do empreendimento da subestação, se faz necessário o uso do licenciamento ambiental. Para que esta atividade ocorra do início até o fim da obra, o empreendimento deve passar por três licenças, as quais são regulamentadas pela Resolução № 237, de 19 de dezembro de 1997, solicitadas de acordo com a fase em que se encontra o empreendimento, sendo elas, licença prévia (LP), licença de instalação (LI) e licença de operação (LO) (BRASIL 1997).

Para o licenciamento prévio do empreendimento uma das exigências do órgão licenciador é a elaboração de estudo ambiental simplificado, no qual apresenta as características técnicas do empreendimento e o diagnóstico ambiental do local (GEO CONSULTORES 2016). Comumente para a realização desta atividade de avaliação dos impactos, as empresas de consultoria utilizam método Ad Hoc, porém demonstram os resultados com a utilização de matrizes simples. Essas matrizes simples tendem a não demonstrar a importância e nem interação entre as atividades realizadas e os impactos ambientais.

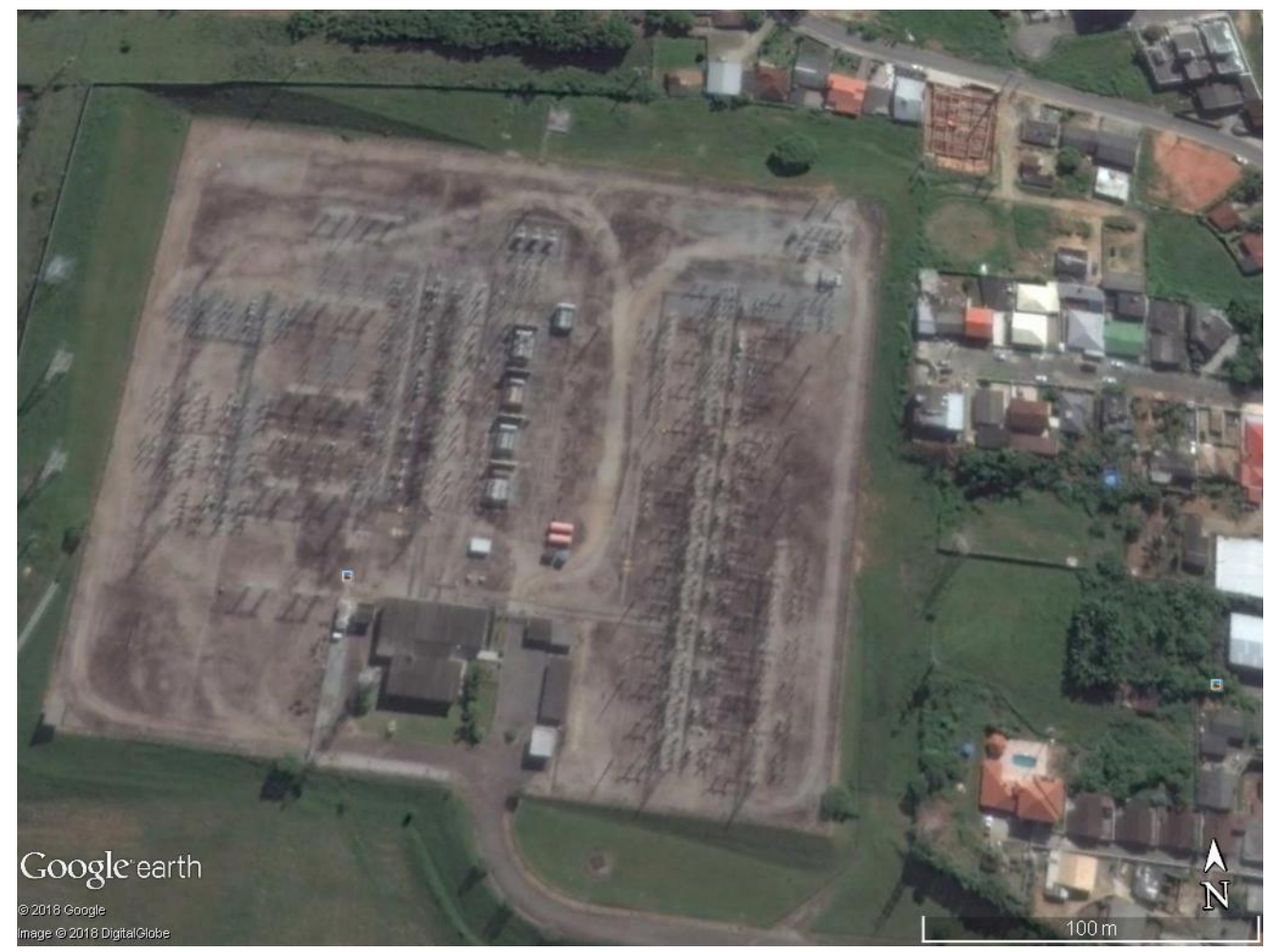

Figura 1. A vista geral da área de estudo da subestação de energia elétrica em Palhoça, SC, foi obtida por meio do Google Earth Pro, imagem do satélite DigitalGlobe com resolução de 1024 x 768, referente ao dia 22 de abril de 2017.

Figure 1. Overview of the study area of the electric energy in Palhoça, SC obtained through Google Earth Pro, image taken from the satellite DigitalGlobe with resolution of 1024 x 768, referring to April 22, 2017. 
Para a implantação da subestação, são previstas diferentes atividades as quais geram impactos e interações diferentes com o meio. Os impactos ambientais são causados ao meio físico, biótico e socioeconômico, conforme a atividade que está sendo realizada. As atividades a serem realizadas no local passarão por duas fases distintas, sendo a primeira de obras civis, que englobam os serviços de terraplanagem, construção de rede de drenagem do terreno, colocação de brita, malha de aterramento, instalação de muros e portão de acesso, entre outros. Posteriormente, a segunda fase de implantação da subestação, consiste na montagem eletromecânica.

No presente estudo de caso, após a instalação, a operação da subestação tem previsão de começar a funcionar com dois transformadores de 20/26,67 MVA. Sendo assim, a subestação operará em barra simples, contando com duas conexões de transformadores completas com chaves, disjuntores e para-raios. O setor de baixa tensão contará com a implantação de seis bays de $13,8 \mathrm{kV}$.

Para a avaliação dos impactos ambientais gerados com a implantação da subestação contrapondo o método simples de matriz geralmente utilizado por empresas de consultoria, devido ser um método de fácil construção de matriz, porém gerando um resultado resumido, foi utilizado o método $A d H o c$ com matrizes de interação (SILVA 1999) e, para a avaliação do grau de importância, foi utilizada a matriz de ponderação de atributos.

O método Ad Hoc permite o envolvimento direto dos interessados, sendo este um ponto positivo do método. Este consiste em brainstorming (debates), por meio da prática de reuniões entre especialistas de diversas áreas, em que os impactos são caracterizados e sumarizados por meio de tabelas e matrizes (PIMENTEL \& PIRES 1992, MORGAN 2017). Este método tem como objetivo derivar uma listagem descritiva dos impactos ambientais prognosticáveis (VALDETARO et al. 2015).

O método de matriz de interação tem por objetivo indicar a existência de impacto ambiental e se possível estabelecer alguma relação entre a ação/atividade (linha) e o fator ambiental (coluna) (VALDETARO et al. 2015). Por meio deste método de interação são estabelecidas as tabelas usadas para identificar a interação entre as atividades do projeto (ação geradora), os aspectos e impactos ambientais (MORRIS \& THERIVEL 1995).

No estudo foram analisadas as atividades referentes às etapas de implantação e operação do empreendimento, identificando-se as ações geradoras (atividades) e os impactos ambientais. Esta relação de causa e efeito, definida por SÁNCHEZ (2013), busca identificar e estabelecer conexões.

Para o presente estudo foram estabelecidas as seguintes definições:

Ação Geradora é toda e qualquer atividade decorrente da implantação do empreendimento, estando inclusas neste conceito as atividades de planejamento, implantação e operação do empreendimento, cada uma com suas subatividades (GEO CONSULTORES 2016).

A Resolução CONAMA no 01 de 23 de janeiro de 1986 define impacto ambiental como qualquer alteração das propriedades físicas, químicas e biológicas do meio ambiente. É causada por qualquer forma de matéria ou energia resultante das atividades humanas que, direta ou indiretamente, afetem a saúde, a segurança e o bem-estar da população, as atividades sociais e econômicas, a biota, as condições estéticas e sanitárias do meio ambiente e a qualidade dos recursos ambientais (BRASIL 1986). É a mudança ocorrida (ou potencial) em determinado parâmetro ambiental em determinado período (WATHERN 1988).

A matriz de ponderação tem como função principal a definição do grau de importância de cada impacto. Uma das etapas para a determinação das matrizes de ponderação é a definição de magnitudes ou valores a serem ponderados, que são definidos de acordo com a importância relativa de cada atributo e critério (AVILA \& GONÇALVES 2010).

Os impactos listados por meio do método Ad Hoc foram avaliados quanto aos atributos: natureza dos impactos; localização e espacialização; fase de ocorrência; incidência; duração; temporalidade; reversibilidade; ocorrência e magnitude. Estes atributos foram classificados conforme escala e receberam valores para ponderação na matriz, para efeito de valoração dos impactos (Figura 2).

A natureza dos impactos classifica os impactos ambientais de forma positiva/benéfica ou de forma negativa/adversa. Os impactos positivos são aqueles que acrescentam benefícios associados a sua ocorrência e poderão ser potencializados com a adoção de medidas específicas pertinentes ao tipo de impacto. Já os impactos negativos poderão ter seus efeitos mitigados, prevenidos, eliminados ou compensados mediante a adoção de medidas específicas.

A localização e Espacialização faz referência à abrangência do impacto frente às áreas de influência definidas neste estudo. Para tanto, foram classificados em localizado na AID - Área de Influência Direta (em torno de $250 \mathrm{~m}$ no entorno da área do empreendimento), localizado na All - Área de Influência Indireta (área delimitada pela bacia hidrográfica de cada local) e difuso na área de influência (que ocorre de forma 
difusa/em diversas direções entre a AID e AII) (GEO CONSULTORES 2016).

\begin{tabular}{|c|c|c|c|c|c|}
\hline \multicolumn{3}{|c|}{ Fase de Ocorrência } & Atributo (valor do atributo) & Escala & Valor Atribuído \\
\hline \multirow{14}{*}{ 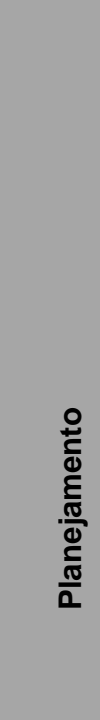 } & \multirow{14}{*}{ 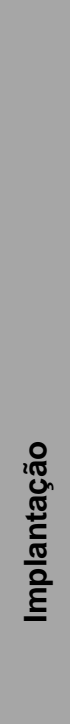 } & \multirow{14}{*}{ 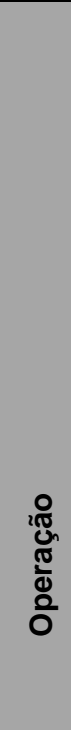 } & \multirow{3}{*}{ Localização/Espacialização (2) } & Localizado na AID & 1 \\
\hline & & & & Localizado na All & 2 \\
\hline & & & & Difuso na área de influência & 3 \\
\hline & & & \multirow{3}{*}{ Duração e Temporalidade (3) } & Temporário e curto prazo & 1 \\
\hline & & & & Cíclico e médio prazo & 2 \\
\hline & & & & Permanente e longo prazo & 3 \\
\hline & & & \multirow{2}{*}{ Reversibilidade (5) } & Reversível & 1 \\
\hline & & & & Irreversível & 2 \\
\hline & & & \multirow{3}{*}{ Ocorrência (3) } & Certo & 3 \\
\hline & & & & Provável & 2 \\
\hline & & & & Improvável & 1 \\
\hline & & & \multirow{3}{*}{ Magnitude (5) } & Baixa & 1 \\
\hline & & & & Média & 2 \\
\hline & & & & Alta & 3 \\
\hline
\end{tabular}

Figura 2. Pesos atribuídos às escalas dos atributos de um determinado impacto para realização de ponderação.

Figure 2. Weights assigned to the attribute scale of a given impact for ponderation.

Duração e temporalidade, indicam o tempo da alteração ambiental. Podem ser temporário e curto prazo, quando determinado impacto tem sua duração finita em um tempo determinado. Podem ser permanente e longo prazo, quando o impacto se mantém após o término da ação geradora; e cíclico e médio prazo, quando o impacto se manifesta em intervalos de tempo ao longo de um período, oriundo de uma ação geradora também recorrente (GEO CONSULTORES 2016).

Reversibilidade indica a possibilidade após o encerramento da ação geradora, o meio alterado retornar à sua condição de origem. Quanto à reversibilidade, um determinado impacto pode ser reversível, quando o meio afetado pelo impacto retorna à sua condição de origem, ou irreversível, quando o meio afetado não retorna mais à sua condição de origem, podendo apenas ser o impacto mitigado ou compensado (GEO CONSULTORES 2016).

A ocorrência indica a probabilidade de determinado impacto realmente ocorrer. Pode ser certa, quando se sabe que determinado impacto sempre ocorre; pode ser provável, quando não há certeza quanto à ocorrência de um determinado impacto; ou pode ser improvável, quando a ocorrência do impacto é incerta e/ou muito pequena (GEO CONSULTORES 2016).

Em relação a magnitude, esta refere-se à grandeza de um impacto em termos absolutos. É considerada a intensidade com que um impacto pode se manifestar, sendo que um impacto pode ser de baixa, média ou de alta magnitude (GEO CONSULTORES 2016).

Por meio da matriz de interação foi possível realizar a ponderação dos valores. Avaliando a pontuação foi possível realizar a classificação do grau de importância para cada impacto, favorecendo a tomada de decisão (Tabela 1).

Para SÁNCHEZ (2013), a ponderação de atributos é o arbítrio entre distintas alternativas de dar pesos a cada um dos atributos selecionados, combinando-os de acordo com uma função matemática predeterminada. Ainda enfatiza sobre a etapa que avalia a importância dos impactos ambientais, sendo que esta é uma das mais difíceis de qualquer estudo de impacto ambiental, pelo motivo de que atribuir maior ou menor grau de importância a uma alteração ambiental depende não só de um trabalho técnico, mas também de um juízo de valor.

Tabela 1. Classificação do grau de importância dos impactos.

Table 1. Degree classification of environmental impact importance.

\begin{tabular}{lll}
\hline Importância & Limite inferior & Limite superior \\
\hline Pequena $(P)$ & 18 & 28 \\
Média $(M)$ & 29 & 39 \\
Grande $(G)$ & 30 & 49 \\
\hline
\end{tabular}




\section{RESULTADOS E DISCUSSÃO}

Como pode ser observado na matriz de ponderação (Figura 3), a maioria dos impactos foram classificados como sendo de pequena importância. Isso se deve principalmente pelos impactos na sua maioria serem reversíveis e de pequena magnitude, como exemplo a alteração da qualidade do ar, aumento do escoamento superficial e erosão e favorecimento a proliferação de vetores.

Entre os impactos positivos pode-se destacar o aumento da oferta de empregos diretos (exemplos: operadores de máquinas e mão de obra braçal) e indiretos (exemplos: restaurantes e alojamentos/hotel) e a melhoria no fornecimento de energia, a qual já estava em estado deficitário principalmente na temporada de verão, momento em que a cidade mais sofre com a atividade de turismo. Em relação aos impactos negativos, a redução da cobertura vegetal no momento da instalação do empreendimento, apresentou maior valor de importância (50 - classificada como de grande importância), sendo considerado o impacto negativo que se deve fornecer maior atenção na coleta de dados e programas de compensação e mitigação.

$\mathrm{Na}$ instalação da subestação, a supressão da vegetação é realizada para que seja possível o lançamento de cabos, a locação e a construção das estruturas (da rede e da própria subestação) e para que haja segurança no momento da energização, evitando posteriores desligamentos da rede e pequenas explosões/incêndios (quando galhos tocam a linha energizada).

Segundo FERREIRA (2011), em estudo de impactos ambientais com linhas de transmissão, verificou que o maior impacto a instalação da linha é a supressão da vegetação nativa, pois além desta interferir em outros impactos, como no deslocamento da fauna, a retirada da vegetação altera as características do habitat, influenciando diretamente no ecossistema local.

PEREIRA (2014), em estudo de impactos ambientais em linhas de transmissão e subestação, também considerou a supressão da vegetação como impacto ambiental negativo, permanente, irreversível, com probabilidade de ocorrência certa, com grande magnitude e grande importância, além de ser capaz de intensificar outros impactos, como o deslocamento da fauna.

Em virtude de ser um impacto (supressão da vegetação) negativo e grande importância, destaca-se a realização de medidas de compensação, as quais tem como função principal restabelecer o equilíbrio dos impactos negativos que foram causados e que não puderam ser evitados e/ou mitigados (SETANI 2015), um exemplo de medida de compensação para a supressão da vegetação que será de responsabilidade da empresa construtora do empreendimento são o Programa de Recuperação de Áreas Degradadas (PRAD), o Programa de Controle de Supressão Vegetal e o Programa de Afugentamento e Resgate Brando de Fauna.

Deve-se atentar também à característica temporária da maioria dos impactos negativos, entre os quais, podem ser citados a alteração da qualidade do ar e da água, aumento do escoamento superficial e erosão, deslocamento da fauna, favorecimento da proliferação de vetores, perturbação da população em torno do traçado. Estes estarão presentes principalmente na fase de implantação do empreendimento, que serão encerrados após a conclusão das obras. O único impacto negativo temporário que estará presente na fase de operação são as falhas/ruínas da linha de transmissão.

Em relação as atividades a serem realizadas na área para a instalação da subestação podem ser citadas: terraplanagem, locação e construção de estruturas, escavações, sistema de drenagem e aterramento, montagens eletromecânicas, mobilização de pessoal e manutenção da subestação. A maioria das atividades serão realizadas no momento de instalação da subestação, sendo esta a fase de maior geração de impactos ambientais em comparação a fase posterior de operação da mesma. Isso ocorre devido a instalação do empreendimento modificar a paisagem, ou seja, é nesta fase que ocorre a supressão da vegetação, o nivelamento do solo e é nesta fase que as atividades que mais afetam os meios físico, biótico e socioeconômico são desenvolvidas, e já na fase de operação a movimentação de pessoas e máquinas é mínima em relação a fase de instalação.

Por meio da matriz de interação, segundo FINUCCI (2010), fica mais evidente relacionar os impactos à ação geradora, sendo que a matriz tem como objetivo relacionar as interações entre as atividades realizadas e os impactos ambientais gerados (Figura 4).

A atividade de terraplanagem, que consiste no desmonte (desaterro ou corte) e no transporte de terras no aterro, foi considerada a atividade de maior impacto. Gerou um grande número de aspectos ambientais favorecendo os impactos como: aumento do escoamento superficial e erosão, alteração da qualidade da água, redução da cobertura vegetal, deslocamento da fauna e ocorrência de processos erosivos.

Em relação aos critérios avaliados, é possível observar que a maioria dos impactos irão ocorrer na Área de Influência Direta (76,92\%), ou seja, próximo à subestação. Estes serão com duração temporária e de curto prazo, sendo consideradas reversíveis, com baixa magnitude e pequena importância (Figura 5). 


\begin{tabular}{|c|c|c|c|c|c|c|c|c|c|c|}
\hline 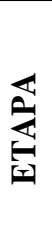 & 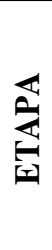 & $\frac{0}{2}$ & IMPACTO & 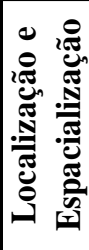 & 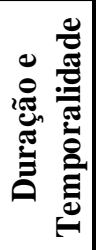 & 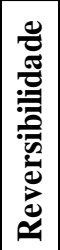 & 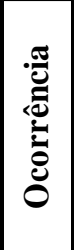 & 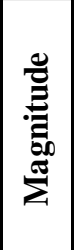 & 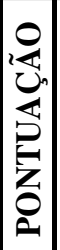 & 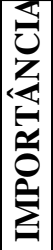 \\
\hline \multirow{8}{*}{ 焉 } & \multirow{8}{*}{ 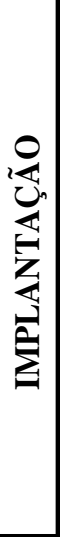 } & \multirow{3}{*}{ 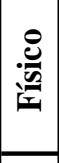 } & Alteração da Qualidade do Ar & $2 * 2$ & $1 * 3$ & $1 * 5$ & $3 * 2$ & $1 * 5$ & 23 & $\mathrm{P}$ \\
\hline & & & Aumento do Escoamento Superficial e Erosão & $1 * 2$ & $1 * 3$ & $1 * 5$ & $2 * 2$ & $1 * 5$ & 19 & $\mathrm{P}$ \\
\hline & & & Alteração da Qualidade da Água & $1 * 2$ & $1 * 3$ & $1 * 5$ & $2 * 2$ & $1 * 5$ & 19 & $\mathrm{P}$ \\
\hline & & \multirow{5}{*}{ 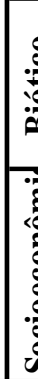 } & Redução da Cobertura Vegetal & $1 * 2$ & $3 * 3$ & $2 * 5$ & $3 * 2$ & $2 * 5$ & 37 & $\mathrm{G}$ \\
\hline & & & Deslocamento de Fauna & $1 * 2$ & $1 * 3$ & $1 * 5$ & $2 * 2$ & $1 * 5$ & 19 & $\mathrm{P}$ \\
\hline & & & Favorecimento a proliferação de vetores & $1 * 2$ & $1 * 3$ & $1 * 5$ & $2 * 2$ & $1 * 5$ & 19 & $\mathrm{P}$ \\
\hline & & & Perturbação da População do Entorno do Traçado & $1 * 2$ & $1 * 3$ & $1 * 5$ & $3 * 2$ & $1 * 5$ & 21 & $\mathrm{P}$ \\
\hline & & & Aumento na Oferta de Empregos & $3 * 2$ & $1 * 3$ & $1 * 5$ & $3 * 2$ & $2 * 5$ & 30 & $\mathrm{M}$ \\
\hline \multirow{5}{*}{ 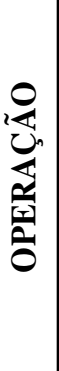 } & \multirow{5}{*}{ 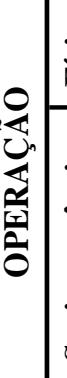 } & 递 & Ocorrência de Processos Erosivos (Operação) & $1 * 2$ & $2 * 3$ & $1 * 5$ & $2 * 2$ & $1 * 5$ & 22 & $\mathrm{P}$ \\
\hline & & $\stackrel{\nexists}{\nexists}$ & Variações dos níveis de campos eletromagnéticos & $1 * 2$ & $3 * 3$ & $2 * 5$ & $2 * 2$ & $1 * 5$ & 30 & $\mathrm{M}$ \\
\hline & & 芯 & $\begin{array}{l}\text { Invasão da faixa de servidão ou praças da linha } \\
\text { de transmissão }\end{array}$ & $1 * 2$ & $2 * 3$ & $1 * 5$ & $1 * 2$ & $1 * 5$ & 20 & $\mathrm{P}$ \\
\hline & &. & Falha/ruína da linha de transmissão & $1 * 2$ & $1 * 3$ & $1 * 5$ & $1 * 2$ & $2 * 5$ & 22 & $\mathrm{P}$ \\
\hline & & ڤ̆ & Melhoria no fornecimento de Energia & $3 * 2$ & $3 * 3$ & $2 * 5$ & $3 * 2$ & $3 * 5$ & 46 & G \\
\hline
\end{tabular}

Figura 3. Matriz de ponderação entre os impactos ambientais gerados na instalação e ampliação da subestação de Palhoça, SC.

Figure 3. Weighting matrix between the environmental impacts generated in the installation and expansion of the electrical substation of Palhoça, SC.

\begin{tabular}{|c|c|c|c|c|c|c|c|c|c|c|c|c|c|c|}
\hline \multicolumn{2}{|c|}{ Classificação de Impactos } & \multicolumn{13}{|c|}{ Impactos ambientais } \\
\hline - & Impacto muito importante & \multicolumn{8}{|c|}{ Instalação } & \multicolumn{5}{|c|}{ Operação } \\
\hline $\mathbf{x}$ & Impacto pouco importante & \multicolumn{3}{|c|}{ Físico } & \multicolumn{3}{|c|}{ Biótico } & \multicolumn{2}{|c|}{ Socioeconômico } & Físico & \multicolumn{4}{|c|}{ Socioeconômico } \\
\hline & Atividades & 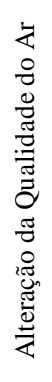 & 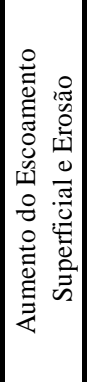 & 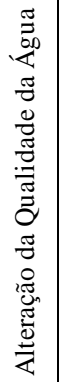 & 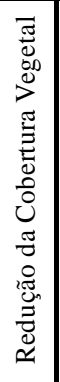 & 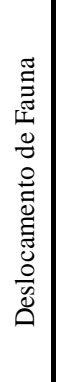 & 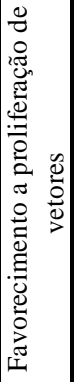 & 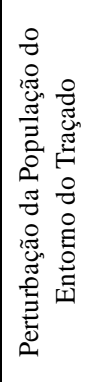 & 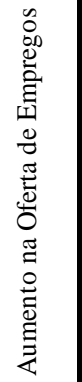 & 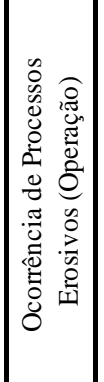 & 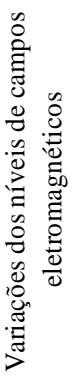 & 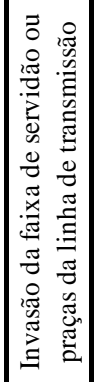 & 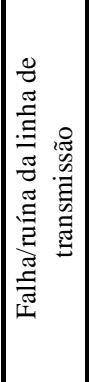 & 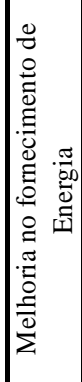 \\
\hline & Terraplanagem & - & - & • & • & • & $\mathbf{x}$ & $\mathbf{x}$ & & - & & $\mathbf{x}$ & & \\
\hline Locaç & e construção de estruturas & & & & & $\mathbf{x}$ & & & $\bullet$ & & & & & - \\
\hline & Escavações & $\mathbf{x}$ & $\mathbf{x}$ & $\mathbf{x}$ & - & - & $\mathbf{x}$ & & & - & & $\mathbf{x}$ & & \\
\hline Sistem & de drenagem e aterramento & & $\mathbf{x}$ & $\bullet$ & & & $\mathbf{x}$ & $\mathbf{x}$ & & & & & & \\
\hline & tagens eletromecânicas & & & & & & & $\mathbf{x}$ & & & $\mathbf{x}$ & $\mathbf{x}$ & $\mathbf{x}$ & \\
\hline & obilização de pessoal & & & & & & & & - & & & & & \\
\hline & uutenção da subestação & $\mathbf{x}$ & & & & $\mathbf{x}$ & & $\mathbf{x}$ & & & $\mathbf{x}$ & & $\mathbf{x}$ & - \\
\hline
\end{tabular}

Figura 4. Matriz de interação entre as atividades realizadas nas fases de instalação e operação da subestação e os impactos ambientais gerados.

Figure 4. Matrix of interaction between the activities carried out during the installation and operation of the substation as well as the environmental impacts generated. 
Em relação à duração e temporalidade, a maioria dos impactos ambientais foram classificados como de duração temporária e de curto prazo, ocorrendo principalmente na fase de implantação da subestação, ou seja, estes impactos após a implantação não serão mais encontrados no local. Entre estes pode ser citado a geração de empregos, que é um impacto positivo, no qual, PEREIRA (2014) ressalta que as vagas, nesse tipo de empreendimento, são para o período de obras, por isso são temporários.

A Localização e Espacialização

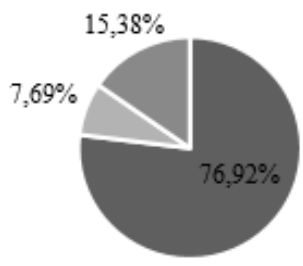

C

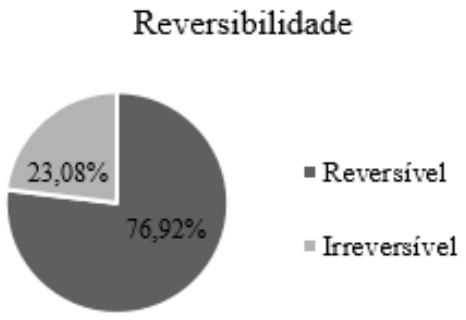

$\mathbf{E}$
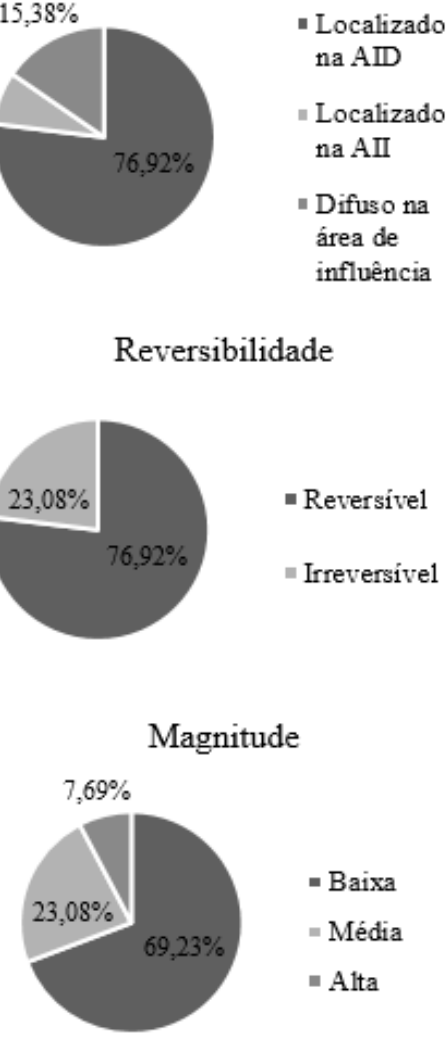

B

Duração e Temporalidade

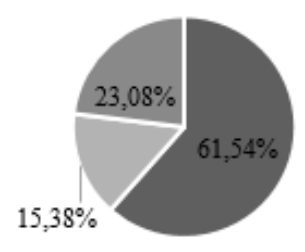

D

Ocorrência

$$
\begin{aligned}
& \text { - Temporário e } \\
& \text { Curto Prazo } \\
& \text { = Cíclico e } \\
& \text { Médio Prazo } \\
& \text { - Permanente e } \\
& \text { Longo Prazo }
\end{aligned}
$$

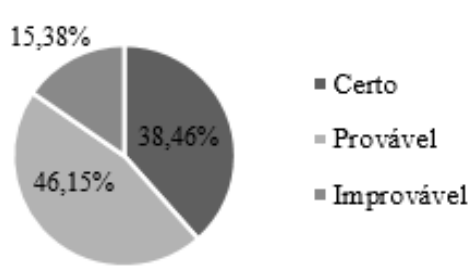

F Importância

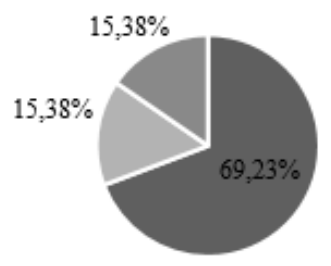

Figura 5. Gráficos em percentagem da classificação dos impactos ambientais para cada critério avaliado. $A=$ Localização e Espacialização; $B=$ Duração e Temporalidade; $C=$ Reversibilidade; $D=$ Ocorrência; $\mathrm{E}=$ Magnitude e $\mathrm{F}=$ Importância.

Figure 5. Percentage graphs of the different environmental impacts evaluated. $A=$ Location and Spatialization; $B=$ Duration and Temporality; $C=$ Reversibility; $D=$ Occurrence; $E=$ Magnitude and $F=$ Importance.

PEREIRA (2014) também menciona que para a construção de uma subestação, torna-se praticamente inevitável a interrupção de vias do local. Esta interrupção pode ser por ação direta dos processos das fases de implantação, no qual há perturbação da população do entorno do traçado a um curto prazo.

Em relação à reversibilidade, a maioria dos impactos são considerados reversíveis. Dentre estes impactos, destaca-se os processos erosivos que, conforme PEREIRA (2014), podem ser revertidos com ações que reestabeleçam a estabilidade do solo e façam sua recomposição.

Outro critério avaliado é a magnitude, cabendo ressaltar, que somente um dos impactos foi considerado de alta magnitude, sendo este a melhoria no fornecimento de energia, que é um impacto que gera a melhoria da qualidade de vida social da população.

Com relação à importância, nove impactos ambientais foram considerados como de pequena importância, sendo que, dois foram considerados de grande importância, a redução da cobertura vegetal e a melhoria no fornecimento de energia.

A redução da cobertura vegetal é de grande importância devido ao seu potencial de gerar outros impactos ambientais, como o aumento do escoamento superficial, erosão do solo e o deslocamento da fauna. De acordo com FERREIRA (2011), a supressão da vegetação altera as características do habitat, influenciando diretamente na fauna local e influência no solo, pois este fica descoberto, aumentando as possibilidades de erosão. 


\section{CONCLUSÃO}

Foram identificados 13 impactos ambientais, derivados da instalação de subestação elétrica, em que oito impactos foram considerados mais significativos pela classificação dos aspectos (alteração da qualidade do ar, aumento do escoamento superficial e erosão, alteração da qualidade da água, redução da cobertura vegetal, deslocamento de fauna, aumento na oferta de empregos temporários, ocorrência de processos erosivos (operação) e melhoria no fornecimento de energia).

Entre os impactos identificados, dois foram classificados como de grande importância, sendo a redução da cobertura vegetal como impacto negativo e a melhoria no fornecimento de energia um impacto positivo.

Considerando a avaliação dos impactos ambientais na instalação da subestação, pode-se afirmar que os métodos alternativos apresentam com mais clareza a magnitude e a interação entre causa e efeito, facilitando a avaliação do empreendimento.

Este estudo pode servir de subsídio para futuros estudos relacionados a avaliação de impactos ambientais, de modo a facilitar a visualização da grandeza dos impactos e a interação, tanto para estudos relacionados à empreendimentos de subestação de energia elétrica, como para a instalação de empreendimentos agroveterinários, quando da adaptação das matrizes.

\section{AGRADECIMENTOS}

Agradecimentos a revisores, colaboradores e agências de fomento (FAPESC).

\section{REFERÊNCIAS}

AVILA SM \& GONÇALVES PB. 2010. Procedimento para determinação das matrizes de ponderação do algoritmo de controle ótimo para um amortecedor de massa híbrido (AMH). Asociación Argentina de Mecánica Computacional XXIX: 1345-1365.

BRASIL. 1986. Resolução CONAMA no 01, de 23 de janeiro de 1986. Que dispõe sobre as definições, as responsabilidades, os critérios básicos e as diretrizes gerais para uso e implementação da Avaliação de Impacto Ambiental como um dos instrumentos da Política Nacional do Meio Ambiente. Diário Oficial [da] República Federativa do Brasil, Brasília, DF, 17 Fev. 1986.

BRASIL. 1987. Resolução CONAMA n 06, de 16 de setembro de 1987. Que dispõe sobre o licenciamento ambiental de obras do setor de geração de energia elétrica. Diário Oficial [da] República Federativa do Brasil, Brasília, DF, 22 Out. 1987.

BRASIL. 1997. Resolução CONAMA n 237 de 19 de dezembro de 1997. Dispõe sobre a revisão e complementação dos procedimentos e critérios utilizados para o licenciamento ambiental. Diário Oficial [da] República Federativa do Brasil, Brasília, DF, 22 Dez. 1997.

BRASIL. 2001. Resolução CONAMA n 279, de 27 de junho de 2001. Que dispõe sobre a necessidade de estabelecer procedimento simplificado para o licenciamento ambiental, dos empreendimentos com impacto ambiental de pequeno porte. Diário Oficial [da] República Federativa do Brasil, Brasília, DF, 29 Jun. 2001.

CAMARGO ASG et al. 2004. Proposta de definição de indicadores de sustentabilidade para geração de energia elétrica. Revista Educação \& Tecnologia 8: 1-21.

DINIZ FB. 2003. Impacto ambiental da emissões sonoras de subestações de energia elétrica na cidade de Curitiba. Dissertação (Mestrado em Engenharia Mecânica). Curitiba: UFPR. $121 \mathrm{p}$.

FERREIRA JB. 2011. Estudo de impactos ambientais e medidas mitigadoras para uma obra de linhas de transmissão de energia elétrica. Trabalho de Conclusão de Curso (Graduação em Agronomia). Florianópolis: UFSC. 48p.

FINUCCI M. 2010. Metodologias utilizadas na avaliação do impacto ambiental para liberação comercial do plantio de transgênicos: uma contribuição ao estado da arte no Brasil. Dissertação (Mestrado em Saúde Pública). São Paulo: USP. 230p.

GEO CONSULTORES. 2016. Estudo Ambiental Simplificado - EAS: subestação Palhoça Caminho Novo. Tubarão: Geo Consultores Engenharia e Meio Ambiente Ltda, 159p.

IBGE. 2012. Manual Técnico da Vegetação Brasileira: Sistema fitogeográfico inventário das formações florestais e campestres, técnicas e manejo de coleções botânicas, procedimentos para mapeamentos. 2.ed. Rio de janeiro: IBGE. 271p. (Manuais Técnicos de Geociências, 1).

LIMA AO de. 2008. Estudo de medidas para controle ambiental em subestações de energia elétrica. Dissertação (Mestrado em Gestão e Políticas Ambientais). Recife: UFPE. 80p.

MORGAN A. 2017. Métodos mais utilizados na avaliação de impactos ambientais. Centro de Produções Técnicas e Editora Ltda. Disponível em: https://www.cpt.com.br/noticias/metodos-mais-utilizados-na-avaliacao-de-impactosambientais. Acesso em: 18 mai. 2017.

MORRIS P \& THERIVEL R. 1995. Methods of Environmental Impact Assessment: the natural and built environmental. 2.ed. Londres: London: UCL Press. 492p.

PEREIRA ALAB. 2014. Análise crítica dos impactos ambientais ocasionados pela linha de transmissão $500 \mathrm{kV}$ Miracema, Sapeaçu e subestações associadas. Trabalho de Conclusão de Curso (Graduação em Engenharia 
Ambiental). Rio de Janeiro: UFRJ. 112p.

PIMENTEL G \& PIRES SH. 1992. Metodologias de avaliação de impacto ambiental: Aplicações e seus limites. Revista de Administração Pública 26: 56-68.

PIRES LFA. 2005. Gestão ambiental da implantação de sistemas de transmissão de energia elétrica. Estudo de caso: Interligação Norte/Sul I. Dissertação (Mestrado em Ciência Ambiental). Niterói: UFF. 142p.

RODRIGUES ERC. 2014. Avaliação do impacto ambiental na implantação do sistema isolado e aéreo de distribuição de energia elétrica na reserva da Sapiranga em Mata de São João, BA. Monografia (Especialização em Gestão Ambiental em Municípios) Medianeira: UFTPR. 56p.

SÁNCHEZ LE. 2013. Avaliação de impacto ambiental - conceitos e métodos. 2.ed. São Paulo: Oficina de Textos. 584p.

SETANI M. 2015. Prevenção, Mitigação e Compensação de Impactos Ambientais de PCHs. Disponível em: http://www.ciclusambiental.net.br/blog/prevencaomitigacaoecompensacaodeimpactosambientaisdepchs. Acesso em: 21 mai. 2017.

SILVA E. 1999. Técnicas de avaliação de impactos ambientais. Viçosa: CPT. 64p. (Vídeo-curso, 199).

SILVA IMP. 2006. Impactos socioambientais da implantação de linha de transmissão de energia elétrica sobre as comunidades extrativistas do babaçu no estado do Maranhão. Dissertação (Mestrado em Gestão e Políticas Ambientais). Recife: UFPE. 103p.

VALDETARO EB et al. 2015. Conjugação dos métodos da matriz de interação e do check-list na avaliação qualiquantitativa de impactos ambientais de um programa de fomento florestal. Revista Árvore 39: 611-622.

WATHERN P. 1988. An introductory guide to EIA. In: WATHERN P. (Org). Environmental impact assessment: theory and practice. London: Unwin Hyman. p. 3-30. 